\title{
SADDLE POINT THEOREM AND NONLINEAR SCALAR NEUMANN BOUNDARY VALUE PROBLEMS
}

\author{
M. N. NKASHAMA
}

(Received 13 May 1991)

Communicated by E. N. Dancer

\begin{abstract}
We are concerned with existence results for nonlinear scalar Neumann boundary value problems $u^{\prime \prime}+g(x, u)=0, u^{\prime}(0)=u^{\prime}(\pi)=0$ where $g(x, u)$ satisfies Carathéodory conditions and is (possibly) unbounded. On the one hand we only assume that the function $(\operatorname{sgn} u) g(x, u)$ is bounded either from above or from below in some function space, and we impose conditions which relate the asymptotic behavior of the function $\int_{0}^{\pi} G(x, u) d x$ (for $|u|$ large) with the first two eigenvalues of the corresponding linear problem (here $G(x, u)=\int_{0}^{u} g(x, s) d s$ is the potential generated by $g$ ). On the other hand we consider cases where the function $(\operatorname{sgn} u) g(x, u)$ is unbounded. The potential $G(x, u)$ is not necessarily required to satisfy a convexity condition. Our method of proof is variational, we make use of the Saddle Point Theorem.
\end{abstract}

1991 Mathematics subject classification (Amer. Math. Soc.): 34 B 15, 34 B 25.

\section{Introduction}

This paper is devoted to the study of existence results for nonlinear scalar Neumann boundary value problems

$$
\begin{aligned}
u^{\prime \prime}(x)+g(x, u(x)) & =0 \text { a.e. in } I, \\
u^{\prime}(0)=u^{\prime}(\pi) & =0
\end{aligned}
$$

where $I=[0, \pi], g: I \times \mathbb{R} \rightarrow \mathbb{R}$ satisfies the Carathéodory conditions (see Section 2) and is (possibly) unbounded.

(C) 1993 Australian Mathematical Society 0263-6115/93 $\$$ A2.00+0.00

This work was supported in part by US National Science Foundation under grant DMS-9006134. 
Let $G(x, u)$ be the potential generated by the function $g$, that is

$$
G(x, u)=\int_{0}^{u} g(x, s) d s .
$$

We impose conditions that relate the asymptotic behavior of the function $\int_{I} G(x, u) d x$ (for $|u|$ large) with the first two eigenvalues of the linear problem

$$
\begin{aligned}
u^{\prime \prime}(x)+\lambda u(x) & =0 \\
u^{\prime}(0)=u^{\prime}(\pi) & =0 .
\end{aligned}
$$

In Section 2, we consider a coercivity condition of Ahmad, Lazer, and Paul's type $([2,11,12])$ below the first eigenvalue, say zero. In Theorem 2.1 we do not assume that the nonlinearity $g(x, u)$ grows at most linearly. We only assume that the function $(\operatorname{sgn} u) g(x, u)$ is bounded from above in some function space. Theorem 2.2 is devoted to the case when no boundedness condition is imposed on the function $(\operatorname{sgn} u) g(x, u)$. In that case we impose a growth condition on the potential $G(x, u)$. In Theorem 2.3 we consider the case when nonresonance occurs below the first eigenvalue. In all our results the potential $G(x, u)$ is not necessarily required to satisfy a convexity condition. The main results of Section 2 are valid for periodic boundary value problem as well. To conclude Section 2 we provide a counterexample which throws more light on the relevance of our results.

Section 3 is devoted to existence conditions at the first two eigenvalues of the problem (1.3). We consider a coercivity condition of Ahmad, Lazer, and Paul's type with respect to the first eigenvalue, complemented by a nonuniform condition with respect to the second eigenvalue of (1.3). In Theorem 3.1 we assume that the function $g(x, u)$ grows at most linearly and that the function ( $\operatorname{sgn} u) g(x, u)$ is only bounded from below in some function space.

Besides the classical real Lebesgue spaces $L^{p}(I)$ and the spaces $C^{p}(I)$ of $p$-times continuously differentiable real valued functions, we shall make use of Sobolev spaces $H^{1}(I)$ and $W^{2,1}(I)$ (see for example, Brézis [3] for definitions and properties).

For each $u \in H^{1}(I)$, we shall write

$$
u(x)=\bar{u}+\tilde{u}(x)
$$

where

$$
\bar{u}=\pi^{-1} \int_{I} u(x) d x \quad \text { and } \quad \tilde{u}(x)=u(x)-\bar{u} .
$$


So, with obvious notations,

$$
H^{1}(I)=\bar{H}^{1}(I) \oplus \tilde{H}^{1}(I) .
$$

For $u \in L^{1}(I)$, we define

$$
u^{+}(x)=\max (u(x), 0) \quad \text { and } \quad u^{-}(x)=\max (-u(x), 0) .
$$

Hence,

$$
u(x)=u^{+}(x)-u^{-}(x)
$$

\section{Existence conditions below the first eigenvalue}

In this section we study the solvability of the Neumann boundary value problem (1.1) where $g$ is a Carathéodory function, that is, $g(\cdot, u)$ is measurable for all $u \in \mathbb{R}, g(x, \cdot)$ is continuous for a.e. $x \in I$, and for each constant $r>0$ there exists a function $f_{r} \in L^{1}(I)$ such that

$$
|g(x, u)| \leq f_{r}(x)
$$

for a.e. $x \in I$, and all $u \in \mathbb{R}$ with $|u| \leq r$.

Let $G(x, u)$ be the potential generated by the function $g$ as defined in (1.2). The following result deals with the case when the function $(\operatorname{sgn} u) g(x, u)$ is bounded from above.

THEOREM 2.1. Suppose there exist functions $A, B \in L^{1}(I)$ and a constant $R \in \mathbb{R}$ with $R>0$ such that

$$
g(x, u) \leq A(x)
$$

for a.e. $x \in I$ and all $u \in \mathbb{R}$ with $u \geq R$,

$$
g(x, u) \geq B(x)
$$

for a.e. $x \in I$ and all $u \in \mathbb{R}$ with $u \leq-R$.

Moreover, assume

$$
\lim _{|u| \rightarrow \infty} \int_{I} G(x, u) d x=-\infty
$$


Then equation (1.1) has at least one solution $u \in W^{2,1}(I)$ that minimizes the functional

$$
\phi(u)=\int_{I}\left[\frac{1}{2}\left|u^{\prime}\right|^{2}-G(x, u)\right] d x
$$

on $H^{1}(l)$.

Conditions (2.2) and (2.3) are used in the literature in connection with the so called Landesman-Lazer condition (see for example, $[8,1]$ ), here they are used along with the more general condition (2.4) of Ahmad, Lazer and Paul's type [2]. On the other hand, regarding (2.4), we do not assume that $g$ is (necessarily) bounded as is usually required in the literature (see for example, $[2,11])$. Also, notice that no convexity assumption is imposed on the potential $G$. The reader is referred to Theorem 3.1 for similar conditions above the first eigenvalue.

PROOF. By (2.1)-(2.3), it follows that there exists a function $b \in L^{1}(I)$ such that

$$
G(x, u) \leq b(x)|u|
$$

for a.e. $x \in I$ and all $u \in \mathbb{R}$.

It is easy to verify that the functional $\phi$, defined by $(2.5)$, is a $C^{1}$-functional on $H^{1}(I)$ since $H^{1}(I)$ is compactly imbedded into $C(I)$ (see for example, [11, pp. 90-94]).

We shall show that $\phi$ is coercive on $H^{1}(I)$, that is,

$$
\phi(u) \rightarrow \infty \quad \text { as } \quad|u|_{H^{\prime}} \rightarrow \infty
$$

which would imply that the Palais-Smale condition is satisfied (see for example, [11, p. 94]).

Assuming that this is the case, we deduce, by [11, Theorem 2.7], that $\phi$ has a minimum at some point $u \in H^{1}(I)$ (see also [11, p. 25]). Since $\phi$ is a $C^{1}$-functional on $H^{1}(I)$, necessarily $\phi^{\prime}(u)=0$, and $u$ is a weak solution to equation (1.1). Therefore, condition (2.1) and a standard regularity result imply that $u \in W^{2,1}(I)$ (see for example, [3, p. 182]).

Now we are going to prove that the functional $\phi$ is coercive on $H^{1}(I)$.

We assume by contradiction that there exists a sequence $\left(u_{n}\right) \subset H^{1}(I)$ with $\left|u_{n}\right|_{H^{1}} \rightarrow \infty$ as $n \rightarrow \infty$ such that

$$
\phi\left(u_{n}\right) \leq c_{1}
$$


for some constant $c_{1}$ and all $n \in \mathbb{N}$.

Set $v_{n}=u_{n} /\left|u_{n}\right|_{H^{\prime}}$. Then, one can find a subsequence relabeled $\left(v_{n}\right)$ and a point $v \in H^{1}(I)$ such that

$$
\left|v_{n}\right|_{H^{1}}=1, \quad v_{n} \rightarrow v \quad \text { in } C(I), \quad v_{n} \rightarrow v \quad \text { in } H^{1}(I) .
$$

By (2.7), one has

$$
\int_{I} \frac{1}{2}\left|v_{n}^{\prime}\right|^{2} d x-\int_{I} \frac{G\left(x, u_{n}\right)}{\left|u_{n}\right|_{H^{\prime}}^{2}} d x \leq \frac{c_{1}}{\left|u_{n}\right|_{H^{1}}^{2}}
$$

which implies, by (2.6), that

$$
\int_{I} \frac{1}{2}\left|v_{n}^{\prime}\right|^{2} d x-\int_{I} b(x) \frac{v_{n}}{\left|u_{n}\right|_{H^{1}}} d x \leq \frac{c_{1}}{\left|u_{n}\right|_{H^{1}}^{2}} .
$$

By going to the limit as $n \rightarrow \infty$, one obtains

$$
v_{n}^{\prime} \rightarrow 0 \text { in } L^{2}(I) .
$$

Therefore, by (2.8), one has $v_{n} \rightarrow v$ in $H^{1}(I), v^{\prime}(x)=0$ for a.e. $x \in I$ which implies that $v \not \equiv 0$, since $v \equiv 0$ would lead to a contradiction with $\left|v_{n}\right|_{H^{1}}=1$.

On the other hand, since $v^{\prime}(x)=0$ for a.e. $x \in I$, we deduce that

$$
v(x)=c
$$

on $I$ for some constant $c \neq 0$. By (2.8), it follows that either $u_{n} \rightarrow \infty$ or $u_{n} \rightarrow-\infty$ uniformly on $I$ as $n \rightarrow \infty$.

Let us assume that $u_{n} \rightarrow \infty$ uniformly on $I$ (the proof for the other case is similar). Setting

$$
u_{n}\left(x_{n}\right)=\min _{I} u_{n}(x),
$$

we obtain $u_{n}\left(x_{n}\right) \rightarrow \infty$. Therefore, by writing $\phi\left(u_{n}\right)$ as

$$
\phi\left(u_{n}\right)=\int_{I}\left[\frac{1}{2}\left|u_{n}^{\prime}\right|^{2}-G\left(x, u_{n}\left(x_{n}\right)\right)-\left(G\left(x, u_{n}(x)\right)-G\left(x, u_{n}\left(x_{n}\right)\right)\right)\right] d x
$$

and using (2.2), it follows that, for sufficiently large $n$,

$$
\begin{aligned}
\phi\left(u_{n}\right) & \geq \int_{I}\left[\frac{1}{2}\left|u_{n}^{\prime}\right|^{2}-G\left(x, u_{n}\left(x_{n}\right)\right)\right] d x-\int_{I} A(x)\left(u_{n}(x)-u_{n}\left(x_{n}\right)\right) d x \\
& \geq \frac{1}{2}\left|u_{n}^{\prime}\right|_{L^{2}}^{2}-\int_{I} G\left(x, u_{n}\left(x_{n}\right)\right) d x-\int_{I} A(x)\left[\left(u_{n}(x)-\bar{u}_{n}\right)-\left(u_{n}\left(x_{n}\right)-\bar{u}_{n}\right)\right] d x ;
\end{aligned}
$$


that is,

$$
\phi\left(u_{n}\right) \geq \frac{1}{2}\left|u_{n}^{\prime}\right|_{L^{2}}^{2}-2|A|_{L^{1}}\left|\tilde{u}_{n}\right|_{C}-\int_{I} G\left(x, u_{n}\left(x_{n}\right)\right) d x .
$$

So, by the Sobolev inequality (see for example, [3, p. 129]) one has

$$
\phi\left(u_{n}\right) \geq \frac{1}{2}\left|u_{n}^{\prime}\right|_{L^{2}}^{2}-2 c_{2}\left|u_{n}^{\prime}\right|_{L^{2}}-\int_{l} G\left(x, u_{n}\left(x_{n}\right)\right) d x
$$

for some constant $c_{2}>0$.

It immediately follows from (2.4) and (2.13) that $\phi\left(u_{n}\right) \rightarrow \infty$ as $n \rightarrow \infty$, thus contradicting (2.7). The proof is complete.

In the next result we are concerned with the case when no boundedness condition is imposed on the function $(\operatorname{sgn} u) g(x, u)$. In that case we impose a growth condition on the potential $G(x, u)$. This condition includes the case when the potential $G(x, u)$ is Lipschitz, that is, when the nonlinearity $g(x, u)$ is bounded $($ see $[2,11])$.

THEOREM 2.2. Suppose there exist functions $\alpha, \gamma, \Gamma \in L^{1}(I)$ such that

$$
|G(x, u)-G(x, v)| \leq \Gamma(x)|u-v|^{2}+\gamma(x)|u-v|+\alpha(x)
$$

for a.e. $x \in I$ and all $u, v \in \mathbb{R}$, where

$$
\Gamma(x) \leq 1 / 2
$$

for a.e. $x \in I$ with strict inequality on a subset of positive measure. Moreover, assume condition (2.4) is fulfilled. Then the conclusion of Theorem 2.1 holds.

PROOF. Under conditions of Theorem 2.2, we will show that the functional $\phi$ is coercive on $H^{1}(I)$.

As in the proof of Theorem 2.1 we assume to the contrary that there exists a sequence $\left(u_{n}\right) \subset H^{1}(I)$ with $\left|u_{n}\right|_{H^{1}} \rightarrow \infty$ as $n \rightarrow \infty$ such that (2.7) is satisfied. By writing $\phi\left(u_{n}\right)$ as

$$
\phi\left(u_{n}\right)=\int_{I}\left[\frac{1}{2}\left|u_{n}^{\prime}\right|^{2}-G\left(x, \bar{u}_{n}\right)\right] d x-\int_{I}\left[G\left(x, u_{n}(x)\right)-G\left(x, \bar{u}_{n}\right)\right] d x
$$

and using (2.14), we have

$$
\phi\left(u_{n}\right) \geq \int_{I}\left[\frac{1}{2}\left|u_{n}^{\prime}\right|^{2}-\Gamma(x)\left|\tilde{u}_{n}\right|^{2}\right] d x-\int_{I} \gamma(x)\left|\tilde{u}_{n}\right| d x-\int_{I} \alpha(x) d x-\int_{I} G\left(x, \bar{u}_{n}\right) d x .
$$


By Sobolev inequality and Lemma 1 in $[9$, p. 339], one gets

$$
\phi\left(u_{n}\right) \geq \delta\left|\tilde{u}_{n}\right|_{H^{1}}^{2}-c_{3}\left|\tilde{u}_{n}\right|_{H^{1}}-|\alpha|_{L^{1}}-\int_{I} G\left(x, \bar{u}_{n}\right) d x
$$

for some constants $\delta>0$ and $c_{3}>0$.

Since $\left|u_{n}\right|_{H^{1}} \rightarrow \infty$ as $n \rightarrow \infty$, it follows that $\left|\bar{u}_{n}\right| \rightarrow \infty$ or $\left|\tilde{u}_{n}\right|_{H^{1}} \rightarrow \infty$ as $n \rightarrow \infty$. Therefore, condition (2.4) and inequality (2.16) imply that $\phi\left(u_{n}\right) \rightarrow \infty$ as $n \rightarrow \infty$. This contradicts (2.7), and the proof is complete.

The final main result of this section provides for nonresonance conditions below the first eigenvalue (see $[4,7,11]$ ). The reader is referred to Theorem 3.1 herein for similar nonresonance conditions at the second eigenvalue.

THEOREM 2.3. Suppose

$$
\limsup _{u \rightarrow \infty} \frac{G(x, u)}{u^{2}} \leq \beta_{+}(x), \quad \limsup _{u \rightarrow-\infty} \frac{G(x, u)}{u^{2}} \leq \beta_{-}(x)
$$

uniformly for a.e. $x \in I$, where $\beta_{+}, \beta_{-} \in L^{1}(I)$ are such that

$$
\beta_{+}(x) \leq 0 \text { and } \beta_{-}(x) \leq 0
$$

for a.e. $x \in I$ with strict inequalities on subsets of I of positive measure. Then the conclusion of Theorem 2.1 holds.

PROOF. Under conditions of Theorem 2.3, we shall show that the functional $\phi$ is coercive on $H^{1}(I)$.

An easy adaptation of the argument used in the proof of $[9$, Lemma 1, pp. 339340] and condition (2.18) imply that there exists a constant $\delta=\delta\left(\beta_{+}, \beta_{-}\right)>0$ such that, for any $u \in H^{1}(I)$,

(2.19) $\psi(u) \equiv \int_{I}\left[\frac{1}{2}\left|u^{\prime}\right|^{2}-\left(\beta_{+}(x)\left(u^{+}\right)^{2}+\beta_{-}(x)\left(u^{-}\right)^{2}\right)\right] d x \geq \delta|u|_{H^{\prime}}^{2}$.

On the other hand, conditions (2.1) and (2.17) imply the existence of a function $\beta \in L^{1}(I)$ such that

$$
G(x, u) \leq\left(\beta_{+}(x)+\frac{\delta}{4}\right) u^{2}+\beta(x)
$$


for a.e. $x \in I$ and all $u \in \mathbb{R}$ with $u \geq 0$,

$$
G(x, u) \leq\left(\beta_{-}(x)+\frac{\delta}{4}\right) u^{2}+\beta(x)
$$

for a.e. $x \in I$ and all $u \in \mathbb{R}$ with $u<0$.

Therefore, for $u \in H^{1}(I)$,

$$
\begin{aligned}
\phi(u) & =\int_{i} \frac{1}{2}\left|u^{\prime}\right|^{2} d x-\int_{u \geq 0} G(x, u) d x-\int_{u<0} G(x, u) d x \\
& \geq \int_{I}\left[\frac{1}{2}\left|u^{\prime}\right|^{2}-\left(\beta_{+}(x)\left(u^{+}\right)^{2}+\beta_{-}(x)\left(u^{-}\right)^{2}\right)\right] d x-\frac{\delta}{2} \int_{I} u^{2} d x-2|\beta|_{L^{1}} .
\end{aligned}
$$

Hence, by (2.19),

$$
\phi(u) \geq \psi(u)-\frac{\delta}{2} \int_{I} u^{2} d x-2|\beta|_{L^{1}} \geq \frac{\delta}{2}|u|_{H^{1}}^{2}-2|\beta|_{L^{1}}
$$

which implies that $\phi$ is coercive on $H^{1}(I)$. The proof is complete.

EXAMPLE 2.1. (A counterexample)

For $c \in \mathbb{R}$ with $|c|>1$ the equation

$$
u^{\prime \prime}(x)+\frac{\cos x}{c+\cos x} u(x)=0, \quad u^{\prime}(0)=u^{\prime}(\pi)=0
$$

has nontrivial solutions of the form

$$
u(x)=A[c+\cos x]
$$

for any $A \in \mathbb{R}$ with $A \neq 0$. Therefore, by the Fredholm alternative, the equation

$$
u^{\prime \prime}(x)+\frac{\cos x}{c+\cos x} u(x)=\cos x, \quad u^{\prime}(0)=u^{\prime}(\pi)=0
$$

(with $|c|>1$ ), has no solution. (Note that $\int_{0}^{\pi}(c+\cos x) \cos x d x \neq 0$.) Moreover, by easy computations and change of variables, one has

$$
\int_{0}^{\pi} \frac{\cos x}{c+\cos x} d x=-2 \int_{0}^{\pi / 2} \frac{\cos ^{2} x}{c^{2}-\cos ^{2} x} d x<0
$$

since $|c|>1$. We deduce two facts: 
(1) The coercivity condition (2.4) alone does not guarantee the existence of solution to equation (1.1).

(2) In the nonresonance case, conditions (2.17)-(2.18) cannot be replaced by a weaker assumption of the type

$$
\limsup _{|u| \rightarrow \infty} \frac{G(x, u)}{|u|^{2}} \leq \Gamma(x)
$$

uniformly for a.e. $x \in I$, where $\Gamma \in L^{1}(I)$ is such that

$$
\int_{0}^{\pi} \Gamma(x) d x<0 .
$$

(Also, clearly condition (2.14) of Theorem 2.2 is not satisfied.)

\section{Existence conditions at the first two eigenvalues}

We shall be concerned with existence results for equation (1.1) under resonance and nonresonance conditions between the first two eigenvalues of (1.3). Throughout this section, we shall assume that the function $g$ satisfies Carathéodory's conditions (see Section 2), and grows at most linearly, that is, there exist a constant $d \geq 0$ and a function $e \in L^{1}(I)$ such that

$$
|g(x, u)| \leq d|u|+e(x)
$$

for a.e. $x \in I$ and all $u \in \mathbb{R}$.

THEOREM 3.1. Suppose

$$
\limsup _{u \rightarrow \infty} \frac{g(x, u)}{u} \leq \Gamma_{+}(x) \text { and } \limsup _{u \rightarrow-\infty} \frac{g(x, u)}{u} \leq \Gamma_{-}(x)
$$

for a.e. $x \in I$ where

$$
0 \leq \Gamma_{+}(x) \leq 1, \quad 0 \leq \Gamma_{-}(x) \leq 1
$$

for a.e. $x \in I$ with

$$
\int_{w>0}\left(1-\Gamma_{+}\right) w^{2} d x+\int_{w<0}\left(1-\Gamma_{-}\right) w^{2} d x>0
$$


for all $w \in \operatorname{Span}\{\cos x\}$ with $w \not \equiv 0$. Moreover, assume there exist functions $A, B \in L^{1}(I)$ and a constant $R \in \mathbb{R}$ with $R>0$ such that

$$
g(x, u) \geq A(x)
$$

for a.e. $x \in I$ and all $u \in \mathbb{R}$ with $u \geq R$,

$$
g(x, u) \leq B(x)
$$

for a.e. $x \in I$ and all $u \in \mathbb{R}$ with $u \leq-R$. Finally, suppose

$$
\lim _{|u| \rightarrow \infty} \int_{I} G(x, u) d x=\infty
$$

where $G$ is the potential defined in (1.2). Then equation (1.1) has at least one solution $u \in W^{2,1}(I)$.

PROOF. Under conditions of Theorem 3.1 we shall prove that the conditions of the Rabinowitz Saddle Point Theorem [11, pp. 24-25] are fulfilled where the Banach space is $H^{1}(I)$ as given in (1.4) and the functional $\phi$ on $H^{1}(I)$ is defined in (2.5). Let

$$
V=\bar{H}^{1}(I), \quad X=\tilde{H}^{1}(I) \quad \text { and } \quad D=\{\bar{u} \in V:|\bar{u}| \leq \rho\} .
$$

We will prove that there exists a constant $\rho>0$ such that

$$
\sup _{\partial D} \phi<\inf _{X} \phi
$$

where $\partial D$ is the boundary of $D$.

On the one hand an elementary adaptation of the argument used in the proof of Lemma 1 of [9, pp. 339-340] and conditions (3.3)-(3.4) imply that there exists a constant $\delta=\delta\left(\Gamma_{+}, \Gamma_{-}\right)>0$ such that, for any $\tilde{u} \in X$,

$$
\psi(\tilde{u}) \equiv \frac{1}{2} \int_{l}\left[\left|\tilde{u}^{\prime}\right|^{2}-\left(\Gamma_{+}(x)\left(\tilde{u}^{+}\right)^{2}+\Gamma_{-}(x)\left(\tilde{u}^{-}\right)^{2}\right)\right] d x \geq \delta|\tilde{u}|_{H^{1}}^{2} .
$$

On the other hand conditions (3.1)-(3.2) imply the existence of a function $\Gamma \in L^{1}(I)$ such that

$$
G(x, u) \leq \frac{1}{2}\left(\Gamma_{+}(x)+\frac{\delta}{2}\right) u^{2}+\Gamma(x)
$$


for a.e. $x \in I$ and all $u \in \mathbb{R}$ with $u \geq 0$,

$$
G(x, u) \leq \frac{1}{2}\left(\Gamma_{-}(x)+\frac{\delta}{2}\right) u^{2}+\Gamma(x)
$$

for a.e. $x \in I$ and all $u \in \mathbb{R}$ with $u<0$.

Therefore, for $\tilde{u} \in X$,

$$
\begin{aligned}
\phi(\tilde{u}) & =\frac{1}{2} \int_{I}\left|\tilde{u}^{\prime}\right|^{2} d x-\int_{\tilde{u} \geq 0} G(x, \tilde{u}) d x-\int_{\tilde{u}<0} G(x, \tilde{u}) d x \\
& \geq \frac{1}{2} \int_{I}\left[\left|\tilde{u}^{\prime}\right|^{2}-\left(\Gamma_{+}(x)\left(\tilde{u}^{+}\right)^{2}+\Gamma_{-}(x)\left(\tilde{u}^{-}\right)^{2}\right)\right] d x-\frac{\delta}{2} \int_{I} \tilde{u}^{2} d x-2|\Gamma|_{L^{\prime}}
\end{aligned}
$$

Hence, by (3.10),

$$
\phi(\tilde{u}) \geq \psi(\tilde{u})-\frac{\delta}{2}|\tilde{u}|_{L^{2}}^{2}-2|\Gamma|_{L^{1}},
$$

that is,

$$
\phi(\tilde{u}) \geq \frac{\delta}{2}|\tilde{u}|_{H^{1}}^{2}-2|\Gamma|_{L^{1}},
$$

which implies that $\phi$ is bounded below on $X$ by $-2|\Gamma|_{L^{1}}$. Moreover, for $\bar{u} \in V$,

$$
\phi(\bar{u})=-\int_{I} G(x, \bar{u}) d x .
$$

Therefore, by using condition (3.7) and inequality (3.13), we obtain the assertion (3.9) for some constant $\rho>0$.

It remains to prove that the functional $\phi$ satisfies the Palais-Smale condition. For this purpose, it suffices, as is easily seen from [11, pp. 94-95], to show that for any sequence $\left(u_{n}\right) \subset H^{1}(I)$ such that $\phi\left(u_{n}\right)$ is bounded and $\phi^{\prime}\left(u_{n}\right) \rightarrow 0$ as $n \rightarrow \infty$, it follows that $\left(u_{n}\right)$ is bounded.

Suppose there exist a sequence $\left(u_{n}\right) \subset H^{1}(I)$ and a constant $c>0$ such that

$$
\begin{cases}\left|u_{n}\right|_{H^{\prime}} \rightarrow \infty & \text { as } n \rightarrow \infty, \\ \left|\phi\left(u_{n}\right)\right| \leq c & \text { for all } n \in \mathbb{N}, \\ \phi^{\prime}\left(u_{n}\right) \rightarrow 0 & \text { as } n \rightarrow \infty\end{cases}
$$

Define the sequence $\left(v_{n}\right) \subset H^{1}(I)$ by

$$
v_{n}=u_{n} /\left|u_{n}\right|_{H^{1}} .
$$


The last assertion in (3.15) implies that

$$
\int_{I}\left[u_{n}^{\prime} w^{\prime}-g\left(x, u_{n}\right) w\right] d x \rightarrow 0 \text { as } n \rightarrow \infty \text { for all } w \in H^{1}(I) .
$$

Therefore

(3.18) $\int_{I}\left[v_{n}^{\prime} w^{\prime}-\left(g\left(x, u_{n}\right) /\left|u_{n}\right|_{H^{\prime}}\right) w\right] d x \rightarrow 0 \quad$ as $n \rightarrow \infty$ for all $w \in H^{1}(I)$.

Since $\left|v_{n}\right|_{H^{\prime}}=1$, by the compact imbedding of $H^{1}(I)$ into $C(I)$ we deduce, passing if necessary to a subsequence relabelled $\left(v_{n}\right)$, that there exists $v \in H^{1}(I)$ such that

$$
v_{n} \rightarrow v \quad \text { in } C(I), \quad v_{n} \rightarrow v \quad \text { in } \quad H^{1}(I), \quad \text { as } n \rightarrow \infty .
$$

On the other hand, by the growth condition (3.1), one has that the sequence $\left(g\left(x, u_{n}\right) /\left|u_{n}\right|_{H^{\prime}}\right)$ is such that

$$
\left|g\left(x, u_{n}\right)\right| /\left|u_{n}\right|_{H^{1}} \leq c_{0}+e(x)
$$

for a.e. $x \in I$ and all $n \in \mathbb{N}$ with $n$ sufficiently large, where $c_{0}$ is some constant.

Hence, by the Dunford-Pettis Theorem (see [3]), the sequence $\left(g\left(x, u_{n}\right) /\left|u_{n}\right|_{H^{1}}\right)$ converges weakly in $L^{1}(I)$. So, by (3.18), we get

$$
\int_{I}\left[v^{\prime} w^{\prime}-K(x) w\right] d x=0 \quad \text { for all } w \in H^{1}(I)
$$

where $K$ is the weak limit (in $\left.L^{1}(I)\right)$ of the sequence $\left(g\left(x, u_{n}\right) /\left|u_{n}\right|_{H^{1}}\right)$.

We claim that $v \not \equiv 0$. Indeed, by conditions (3.1)-(3.6), there exist functions $a, b, h \in L^{1}(I)$ such that

$$
a(x)|u|-b(x) \leq G(x, u) \leq 2|u|^{2}+h(x)
$$

for a.e. $x \in I$ and all $u \in \mathbb{R}$. Hence,

$$
\frac{a(x)}{\left|u_{n}\right|_{H^{1}}} v_{n}-\frac{b(x)}{\left|u_{n}\right|_{H^{1}}^{2}} \leq \frac{G\left(x, u_{n}\right)}{\left|u_{n}\right|_{H^{1}}^{2}} \leq 2 v_{n}^{2}+\frac{h(x)}{\left|u_{n}\right|_{H^{1}}^{2}} .
$$

Now, if $v \equiv 0$, then by the first assertion in (3.19), one deduces $v_{n} \rightarrow 0$ uniformly. So, by (3.22),

$$
\int_{I} \frac{G\left(x, u_{n}\right)}{\left|u_{n}\right|_{H^{1}}^{2}} d x \rightarrow 0 \quad \text { as } n \rightarrow \infty,
$$


which would imply, by the second assertion in (3.15), that $v_{n}^{\prime} \rightarrow 0$ in $L^{2}(I)$. Thus, $v_{n} \rightarrow 0$ in $H^{1}(I)$ as $n \rightarrow \infty$. This contradicts the fact that $\left|v_{n}\right|_{H^{1}}=1$. Therefore $v \not \equiv 0$.

Let us define the function $k_{v}$ by

$$
k_{v}(x)= \begin{cases}K(x) / v(x) & \text { if } v(x) \neq 0 \\ 0 & \text { otherwise }\end{cases}
$$

Then (3.21) becomes

$$
\int_{I}\left[v^{\prime} w^{\prime}-k_{v}(x) v w\right] d x=0 \text { for all } w \in H^{1}(I),
$$

that is, $v$ is a weak solution to the problem

$$
\begin{aligned}
z^{\prime \prime}+k_{v}(x) z & =0 \\
z^{\prime}(0)=z^{\prime}(\pi) & =0 .
\end{aligned}
$$

By standard regularity results [3, pp. 139-140], it follows that $v \in W^{2,1}(I)$, and that

$$
\begin{aligned}
v^{\prime \prime}(x)+k_{v}(x) v(x) & =0 \text { a.e. in } I, \\
v^{\prime}(0)=v^{\prime}(\pi) & =0 .
\end{aligned}
$$

Define the functions $k_{v}^{+}$and $k_{v}^{+}$by

$$
k_{v}^{+}(x)= \begin{cases}k_{v}(x) & \text { if } v(x)>0, \\ 0 & \text { otherwise }\end{cases}
$$

and

$$
k_{v}^{-}(x)= \begin{cases}k_{v}(x) & \text { if } v(x)<0 \\ 0 & \text { otherwise. }\end{cases}
$$

Equation (3.23) becomes

$$
\begin{aligned}
v^{\prime \prime}+k_{v}^{+}(x) v^{+}-k_{v}^{-}(x) v^{-} & =0 \text { a.e. in } I, \\
v^{\prime}(0)=v^{\prime}(\pi) & =0 .
\end{aligned}
$$

By using the definition of the functions $k_{v}^{+}, k_{v}^{-}$, inequalities (3.2), (3.5)-(3.6), and properties of liminf and limsup, it follows that for a.e. $x \in I$,

$$
\begin{aligned}
& 0 \leq k_{v}^{+}(x) \leq \Gamma_{+}(x), \\
& 0 \leq k_{v}^{-}(x) \leq \Gamma_{-}(x) .
\end{aligned}
$$


By multiplying (3.23) with $\bar{v}-\tilde{v}$, integrating over $I$, using inequalities (3.25) and (3.10), and the property of the eigenfunction associated with the first eigenvalue of equation (1.3), it follows that $v(x)=c_{1}$ for some constant $c_{1} \neq 0$. We shall assume $c_{1}>0$. (The proof for the case $c_{1}<0$ is similar.)

Since $v_{n} \rightarrow c_{1}$ uniformly on $I$ as $n \rightarrow \infty$, it immediately follows from (3.16) that

$$
u_{n} \rightarrow \infty \text { uniformly on } I \text { as } n \rightarrow \infty,
$$

which implies that there exists $n_{0} \in \mathbb{N}$ such that, for $n \geq n_{0}$,

$$
u_{n}(x) \geq R \text { for all } x \in I .
$$

So, by (3.5), $g\left(x, u_{n}(x)\right) \geq A(x)$ for a.e. $x \in I$ when $n \geq n_{0}$.

Therefore

$$
\int_{g \leq 0} g\left(x, u_{n}(x)\right) d x \geq c_{2}
$$

for some constant $c_{2}$.

On the other hand, by (3.17) with $w=1$, one has

$$
\left|\int_{I} g\left(x, u_{n}(x)\right) d x\right| \rightarrow 0 \text { as } n \rightarrow \infty .
$$

Hence, by combining (3.28) and (3.29), one gets

$$
\int_{I}\left|g\left(x, u_{n}(x)\right)\right| d x \leq c_{3}
$$

for some constant $c_{3}$.

Since $\phi^{\prime}\left(u_{n}\right) \rightarrow 0$ as $n \rightarrow \infty$, it follows that, for some constant $c_{4}>0$,

$$
\left|\phi^{\prime}\left(u_{n}\right) w\right| \leq c_{4}|w|_{H^{1}} \quad \text { for all } \quad w \in H^{1}(I) .
$$

By inequalities (3.30)-(3.31), one has

$$
\begin{aligned}
c_{4}\left|\tilde{u}_{n}\right|_{H^{\prime}} & \geq\left|\phi^{\prime}\left(u_{n}\right) \tilde{u}_{n}\right|=\int_{I}\left[\left|\tilde{u}_{n}^{\prime}\right|^{2}-g\left(x, u_{n}(x)\right) \tilde{u}_{n}(x)\right] d x \\
& \geq \int_{I}\left|\tilde{u}_{n}^{\prime}\right|^{2} d x-c_{3}\left|\tilde{u}_{n}\right|_{C} .
\end{aligned}
$$

Using the continuous imbedding of $H^{1}(I)$ into $C(I)$, one gets

$$
c_{4}\left|\tilde{u}_{n}\right|_{H^{1}} \geq\left|\tilde{u}_{n}^{\prime}\right|_{L^{2}}^{2}-c_{5}\left|\tilde{u}_{n}\right|_{H^{1}}
$$


for some constant $c_{5}>0$. So, by the Poincaré inequality, it follows that

$$
\left|\tilde{u}_{n}\right|_{H^{1}} \leq c_{6}
$$

for some constant $c_{6}>0$.

Set

$$
u_{n}\left(x_{n}\right)=\min _{I} u_{n}(x)
$$

Then, by (3.26),

$$
u_{n}\left(x_{n}\right) \rightarrow \infty \text { as } n \rightarrow \infty .
$$

which also shows that (for $n$ sufficiently large)

$$
u_{n}\left(x_{n}\right) \geq R \text {. }
$$

On the other hand,

$$
\begin{aligned}
\int_{I} G\left(x, u_{n}(x)\right) d x & =\int_{I} G\left(x, u_{n}\left(x_{n}\right)\right) d x+\int_{I}\left[G\left(x, u_{n}(x)\right)-G\left(x, u_{n}\left(x_{n}\right)\right)\right] d x \\
& =\int_{I} G\left(x, u_{n}\left(x_{n}\right)\right) d x+\int_{I}\left[\int_{u_{n}\left(x_{n}\right)}^{u_{n}(x)} g(x, s) d s\right] d x .
\end{aligned}
$$

So, by assumption (3.5), one has

$$
\int_{I} G\left(x, u_{n}(x)\right) d x \geq \int_{I} G\left(x, u_{n}\left(x_{n}\right)\right) d x+\int_{I}\left(u_{n}(x)-u_{n}\left(x_{n}\right)\right) A(x) d x .
$$

By using the fact that $u_{n}(x)-u_{n}\left(x_{n}\right)=u_{n}(x)-\bar{u}_{n}+\bar{u}_{n}-u_{n}\left(x_{n}\right)$, and the imbedding of $H^{1}(I)$ into $C(I)$, one deduces that

$$
\int_{I} G\left(x, u_{n}(x)\right) d x \geq \int_{I} G\left(x, u_{n}\left(x_{n}\right)\right) d x-c_{7}\left|\tilde{u}_{n}\right|_{H^{1}}|A|_{L^{1}}
$$

for some constant $c_{7}>0$. This implies, by (3.32), that

$$
\int_{I} G\left(x, u_{n}(x)\right) d x \geq \int_{I} G\left(x, u_{n}\left(x_{n}\right)\right) d x-c_{8}
$$

for some constant $c_{8}>0$. Therefore, by (3.34), (3.36), and assumption (3.7), it follows that

$$
\int_{I} G\left(x, u_{n}(x)\right) d x \rightarrow \infty \text { as } n \rightarrow \infty .
$$


Moreover, by (3.32),

$$
\phi\left(u_{n}\right)=\int_{I}\left[\frac{1}{2}\left|u_{n}^{\prime}(x)\right|^{2}-G\left(x, u_{n}(x)\right)\right] d x \leq c_{6}^{2}-\int_{I} G\left(x, u_{n}(x)\right) d x .
$$

So, by (3.37),

$$
\phi\left(u_{n}\right) \rightarrow-\infty \text { as } n \rightarrow \infty,
$$

contradicting the second assertion in (3.15). The proof is complete.

EXAMPLE 3.1. Let

$$
g(x, u)=p(x, u) u \sin ^{2} u+a \cos u+h(x)
$$

where $a \in \mathbb{R}, h \in L^{1}(I)$, and $p: I \times \mathbb{R} \rightarrow \mathbb{R}$ is defined by

$$
p(x, u)= \begin{cases}1 & \text { for } x \in I \text { and } u \in \mathbb{R} \text { with } u \geq 0, \\ \Gamma_{-}(x) & \text { for } x \in I \text { and } u \in \mathbb{R} \text { with } u<0\end{cases}
$$

We suppose that $\Gamma_{-}(x)$ is such that

$$
0 \leq \Gamma_{-}(x) \leq \frac{1}{2} \text { for a.e. } x \in I
$$

with $\Gamma_{-}(x)>0$ on a subset of $I$ of positive measure.

It is easily checked that the potential generated by $g$ is given by

$$
G(x, u)=\frac{p(x, u)}{2} u^{2}-\frac{p(x, u)}{2} u \sin 2 u-\frac{p(x, u)}{4} \cos 2 u+a \sin u+h(x) u .
$$

Therefore, by Theorem 3.1 herein, equation (1.1) has at least one solution for every $h \in L^{1}(I)$.

REMARK 3.1. Theorem 3.1 may be related to a result in [6] where the periodic problem is considered. Both results (as others in the literature $[1,6,11,12]$ and references therein) rely upon the Saddle Point Theorem [11]. However, in verifying the Palais-Smale condition, it is important to note that the approach used in [6] does not seem to work for Neumann boundary value problems; while the one developed herein does apply, in a natural way, to the periodic boundary value problem and provides for a more general result as illustrated by Example 3.1 above. 


\section{Acknowledgement}

The author wishes to thank the referee for his careful reading of the manuscript.

\section{References}

[1] S. Ahmad and A. C. Lazer, 'Critical point theory and a theorem of Amaral and Pera', Boll. Un. Mat. Ital. B3 (1984), 583-598.

[2] S. Ahmad, A. C. Lazer and J. L. Paul, 'Elementary critical point theory and perturbation of elliptic boundary value problems at resonance', Indiana University Math. J. 25 (1976), 933-944.

[3] H. Brézis, Analyse fonctionnelle: Théorie et applications, $2^{e}$ tirage (Masson, Paris, 1987).

[4] D. G. de Figueiredo and J. P. Gossez, 'Nonresonance below the first eigenvalue for a semilinear elliptic problem', Math. Ann. 281 (1988), 589-610.

[5] A. Fonda and J. P. Gossez, 'Semicoercive variational problems at resonance: An abstract approach', preprint, 1988.

[6] A. Fonda and D. Lupo, 'Periodic solutions of second order ordinary differential equations', Séminaire mathématique (nouvelle série), (Université de Louvain, Rapport $n^{\circ}$ 127, Mars 1988).

[7] A. Hammerstein, 'Nichtlineare Integralgleichugen nebst Anwendugen', Acta Math. 54 (1930), 117-176.

[8] R. Iannacci and M. N. Nkashama, 'Unbounded perturbations of forced second order ordinary differential equations at resonance', J. Differential Equations 69 (1987), 289-309.

[9] J. Mawhin and J. R. Ward, Jr., 'Periodic solutions of some forced liénard differential equations at resonance', Arch. Math. (Basel) 41 (1983), 337-351.

[10] J. Mawhin, J. R. Ward, Jr. and M. Willem, 'Variational methods and semi-linear elliptic equations', Arch. Rational Mech. Anal. 95 (1986), 269-277.

[11] P. H. Rabinowitz, Minimax methods in critical point theory with applications to differential equations, CBMS Regional Conf. Ser. in Math., 65 (American Math. Soc., Providence, 1986).

[12] M. Ramos and L. Sanchez, 'Variational elliptic problems involving noncoercive functionals', Proc. Royal Soc. Edinburgh Sect. A 112 (1989), 177-185.

University of Alabama at Birmingham

Birmingham, Alabama 35294

U.S.A. 